\title{
Quaderni
}

QUADERN I Communication, technologies, pouvoir

76 | Automne 2011

Les promesses de la biodiversité

\section{La plainte comme un droit? Médiation, politiques publiques et droits des patients}

Fabrizio Cantelli

\section{OpenEdition}

Édition électronique

URL : http://journals.openedition.org/quaderni/137

DOI : 10.4000/quaderni.137

ISSN : 2105-2956

Éditeur

Les éditions de la Maison des sciences de l'Homme

Édition imprimée

Date de publication : 1 septembre 2011

Pagination : 101-109

Référence électronique

Fabrizio Cantelli, «La plainte comme un droit ? Médiation, politiques publiques et droits des patients », Quaderni [En ligne], 76 | Automne 2011, mis en ligne le 01 septembre 2014, consulté le 19 avril 2019.

URL : http://journals.openedition.org/quaderni/137; DOI : 10.4000/quaderni.137 


\section{Politique}

\section{la plainte \\ comme un \\ droit? \\ Médiation, \\ politiques \\ publiques et \\ droits des \\ patients}

Fabrizio

Cantelli

Chargé de recherches F.N.R.S. Université Libre de Bruxelles
Le droit du citoyen à introduire une plainte à l'égard des autorités publiques se retrouve dans la plupart des États. Mais une plainte est-elle un droit garanti et assuré comme un autre ? De quel type de politique est-elle le nom ? Quels sont les limites et les problèmes une fois que le droit de se plaindre concerne un patient, patient qui déploie une critique sur des manquements et des lacunes des professions de la santé ?

La plainte en tant que droit du patient s'inscrit dans un contexte de modernisation des services et d'évaluation des prestations, de proximité avec les citoyens et de respect de ses droits. Un tel droit de se plaindre ne concerne pas que les institutions de santé. En effet, le citoyen dispose d'un tel droit face à de nombreuses institutions, que ce soit au niveau des administrations locales, régionales, nationales (services publics des finances) et européennes (ombudsman européen) sans oublier les transports en commun et les chemins de fer.

Notre contribution porte sur le cas belge et examine particulièrement le droit de se plaindre tel qu'il figure dans la loi sur le droit des patients (2002). À l'instar d'autres États, dont la France qui a introduit une législation sur la participation des patients aux ressorts incertains (Lascoumes, 2007), la Belgique a fait de la plainte une catégorie politique. Après avoir discuté trois travaux sur la plainte, et pointé certaines de leurs limites, nous défendons notre hypothèse selon laquelle la plainte figure en tant que droit et en tant qu'appui d'une politique publique. En se basant sur une enquête de terrain menée sur le cas belge, nous montrons dans un second temps que les appuis d'une telle politique demeurent pourtant largement incertains. 


\section{Premier bilan des travaux sur la plainte}

Une première série de travaux a procédé à une mise en perspective socio-historique de la plainte comme souffrance. J-F Laé (1996) a offert un tableau précis de la genèse des plaintes dans plusieurs domaines au début des années 1900 (accidents au travail, alcool au voltant, etc.). La plainte est ici inscrite dans un contexte qui contraste avec la référence actuelle au plein exercice d'une citoyenneté et à la qualité des services. L'hypothèse défendue par J-F. Laé est la suivante : la plainte, individuelle ou collective, existe dans le domaine public une fois qu'elle parvient à formuler un caractère insupportable. Et c'est cet insupportable qui va ensuite réorganiser les catégories juridiques, administratives et politiques afin de réparer le mal. Dans la même lignée, d'autres travaux (Foli, 2008) ont porté sur la plainte comme souffrance mais cette fois en l'inscrivant dans le contexte de la bureaucratie et de ses transformations.

Une deuxième série de travaux a exploré la plainte en partant des capitaux détenus par les personnes. J. Peneff (2000) a étudié la forme écrite d'une quarantaine de plaintes émises par des patients dans un service d'urgence en France et a dressé une typologie du profil des patients plaignants : le patient issu de classes populaires et le patient des classes moyennes ou supérieures. La plainte ici constitue donc un vecteur aggravant les inégalités sociales. L'hypothèse défendue est la plainte en tant que révélateur d'un consumérisme dominant les sociétés contemporaines: «Dans ce corpus de lettres se manifeste le consumérisme médical, forme d'exigence et d'attente qui étonne beaucoup les professionnels de santé qui semblent découvrir ces clients insatisfaits qui les inondent de demandes et de plaintes. L'offre médicale, dans le cadre de la pluri-spécialité, de la concurrence et de la rivalité public-privé, a modelé sur le long terme un ensemble d'attitudes chez les consommateurs qui ont dégénéré en exigences et réclamations. » (Peneff, 2000, p. 162).

Une troisième série de travaux, ancrés dans la littérature anglo-saxonne sur les systèmes de santé et les professionnels de la santé, a porté sur les relations entre patients plaignants, médecins et gestionnaires de plaintes. L. Mulcahy (2003), à partir d'une enquête de terrain, a analysé les procédures et les intervenants en relation avec la gestion de plaintes émises par les patients. Ces travaux, qui complètent d'autres études anglaises (Nettleton \& Harding, 1994 ; Allsop 1994), ou japonaises (Yajima \& Takayanagi, 2002), ont permis d'approfondir une critique interne du système du National Health Service anglais (NHS) et de ses problèmes.

Ces trois types de travaux montrent que la plainte est une catégorie qui a été longuement travaillée à la fois dans une démarche historique, dans des études sociologiques et dans des travaux mêlant droit et science politique. Toutefois, certaines questions demeurent irrésolues et certains aspects encore inexplorés. De fait, les questions posées dans les premières lignes de cette contribution restent faiblement élucidées : une plainte estelle un droit garanti et assuré comme un autre ? De quel type de politique est-elle le nom ? Les travaux socio-historiques restituent l'historicité de la plainte et sa congruence avec un contexte institutionnel. Cependant, ils n'interrogent pas en propre la plainte comme droit et comme poli- 
tique. Les typologies rendent compte de l'inégale distribution de la capacité à se plaindre parmi les patients - et complètent les études déjà anciennes sur les patients et leurs capacités faire un procès (Press, 1984). En cela, elles arment la critique tant sociologique que politique sur les inconséquences des procédures en place autour de la plainte des patients. On retrouve toutefois un double angle mort d'abord sur l'ensemble des instruments, règles et acteurs qui font que la plainte est une politique et ensuite sur la plainte réduite à une visée consumériste et négligeant par là toute prétention à la justice. Les travaux anglo-saxons rendent davantage justice à l'environnement de la plainte, aux institutions et aux acteurs qui la formalisent. Mais leur abord formaliste de la plainte et l'arrière-plan juridico-administratif privilégié ne parviennent que faiblement à étudier la dimension politique. Notre enquête entend ouvrir ces chantiers.

\section{La plainte du patient comme droit et comme politique}

En Belgique, on peut repérer la plainte en tant que droit lors du vote de la loi sur le droit des patients en 2002, qui constitue la première tentative d'uniformisation des droits du patient. D'une part, cette loi précise et formalise plusieurs catégories de droits : service de qualité (article 5), protection de la vie privée (article 10), information sur l'état de santé (article 7), droit au consentement pour le patient (article 8) - la plupart des articles étant la continuité d'autres textes. D'autre part, elle innove en consacrant les droits relatifs au dossier du patient (article 9) et en créant la fonction de médiateur « droit des patients » (article 11) en charge de dialoguer avec le patient insatisfait, et le cas échéant de gérer les plaintes des patients par rapport au non respect de ces droits. Enfin, les plaintes font l'objet d'un rapport destiné à l'hôpital et à la Commission fédérale « droits du patient », présidée par un professeur d'université, qui se compose des représentants des Mutualités (reprises sous la catégorie " organismes assureurs »), des associations de patients, des praticiens professionnels et des hôpitaux disposant chacun de quatre membres effectifs et de quatre représentants suppléants - les médiateurs ne sont pas présents, même si les associations de médiateurs ont été entendues lors d'une séance.

La description du dispositif se formant autour de la plainte du patient donne déjà à penser la présence d'un droit et la forme d'une politique. La plainte du patient apparaît reliée à trois éléments : la plainte comme opérateur de justice car portant sur des droits bafoués et la possibilité de leur assurer un caractère opérationnel (Singer, $1993)$; la plainte comme innovation sociale car traitée et gérée par des nouveaux professionnels incarnés par les médiateurs droit des patients ; la plainte comme instrument d'évaluation de la loi et de la médiation car faisant l'objet d'un rapport annuel (par institution) opérant une mise en commun des plaintes dans des conditions fixées par l'arrêté royal du 8 juillet 2003 (article 9) : "Chaque année, le médiateur rédige un rapport avec un relevé du nombre de plaintes, l'objet des plaintes et le résultat de ses actes pendant l'année civile précédente. Les difficultés rencontrées par le médiateur dans l'exercice de sa mission et les recommandations éventuelles pour y remédier peuvent également y être reprises. En outre, le rapport annuel mentionne les recommandations du médiateur, et ce compris celles visées à l'ar- 
ticle 11 de la loi relative aux droits du patient, ainsi que la suite y réservée. Le rapport ne peut contenir des éléments par lesquels une des personnes physiques concernées par le traitement de la plainte pourrait être identifiée. \$2. Le rapport annuel visé au $\$ 1{ }^{\text {er }}$ est transmis au plus tard dans le courant du quatrième mois de l'année civile qui suit : $1^{\circ}$ au gestionnaire, au médecin chef, à la direction et au conseil médical de l'hôpital; $2^{\circ}$ à la Commission fédérale "Droits du patient" comme visée à l'article 16 de la loi relative aux droits du patient. Le rapport annuel doit pouvoir être consulté à l'intérieur de l'hôpital par le médecin-inspecteur compétent.»

La plainte ici brièvement décrite est particulière car codifiée et donc assurée par le droit. Toutefois, la Belgique a connu également une série d'initiatives militantes et politiques autour de la plainte appréhendée comme un appui critique de la médecine et du système de santé. La mise à l'agenda de la plainte remonte à des débats parlementaires épars dans les années 1970 et à la mobilisation d'acteurs médicaux progressistes. Créé en 1964, le Groupe d'Etude pour une Réforme de la Médecine publie en 1971 plusieurs travaux, notamment sur la participation des patients et se veut critique à l'égard de l'Ordre des médecins et des syndicats médicaux. L'association PICA, fondée en 1973, militera pour le droit des patients en psychiatrie. En 1979, l'Algemene Bond van Patienten, une association extérieure aux Mutualités, succède à un groupe d'assurés sociaux, ayant fondé l'Antwerpse Gezondheits-komitee au début des années 1970, comprenant des médecins et des comités d'affiliés considérés comme proches d'un parti de gauche radicale, le Partij Voor De Arbeiders. L'ABP politisera la question de la plainte des patients en l'articulant avec une critique des Mutualités, accusées de se taire face aux intérêts des médecins et des hôpitaux - les Mutualités font partie intégrante du modèle social belge de concertation et illustre le rôle des piliers (socialiste, catholique et libéral) dans la société belge. L'ABP va traiter les plaintes des patients et en évaluer la recevabilité et le caractère fondé. Ce travail va être présenté lors d'un congrès à Anvers intitulé «L'histoire des 1001 plaintes ». Le droit de plainte du patient, à l'instar d'autres mesures liées aux droits du patient, sera l'objet d'une proposition de loi en 1997, renouvelée en 1999 et en 2000.

\section{La plainte du patient : Quel droit pour quelle politique?}

Le lecteur pensera que la description du cas belge est restée sommaire. Pourtant, l'analyse de la plainte en tant que droit et politique (et la portée critique qui en ressort) ne peuvent se déployer qu'à partir du moment où l'enquêteur approfondit son regard. La plainte du patient et sa qualification en tant que droit et politique nous paraît d'autant plus sensible et problématique à mesure que l'on s'en approche comme une expérience, plus particulièrement l'expérience d'un environnement. La gamme des problèmes et des promesses liés au droit de se plaindre en tant que patient en ressort plus finement. En effet, plusieurs travaux ethnographiques (Bosk, 2003 ; Millman, 1977) montrent comment la possibilité d'expression d'un reproche, d'un manquement, d'une critique est tue et empêchée par les proches, par le patient mais aussi par le personnel infirmier, médical et plus largement par les conditions et le rythme propres de l'environnement hospitalier 
(interactions furtives, surcharge de travail, etc.). D'autre part, la démarche d'une plainte formelle (et la prise de contact avec le médiateur) est repoussée à l'extrême par les patients et l'entourage dans la mesure où l'on est encore soigné par le personnel de l'hôpital et en son sein (dépendance) et où l'on cherche à éviter à ajouter aux plaintes en tant que symptôme au statut complexe (Dodier, 1993) liées à l'hospitalisation une gamme de problèmes relationnels (évitement du stigmate du patient problématique). Nous allons à présent spécifier le cas belge.

Si la plainte est considérée comme un droit et une politique, encore faut-il poursuivre l'enquête sur les ressorts de ce droit et de cette politique pour en situer la portée et cerner les effets. Les trois traits caractérisant la plainte du patient en Belgique demandent ainsi à être discutés de manière plus précise.

La dimension d'opérateur de justice, d'abord. La plainte apparaît en tant que signalement d'un droit devenu problématique car non respecté. La plainte s'adosse donc à la transgression de droits énumérés plus haut tels que le droit au libre choix du praticien professionnel, le droit à des prestations de soins de qualité, ou le droit à l'information sur l'état de santé. Cette dimension d'opérateur de justice, également analysée dans d'autres contextes notamment au Québec (Clément \& Gagnon, 2006), a un potentiel démocratique mais qui bute sur plusieurs questions. Le texte sur le droit des patients est peu connu par le milieu hospitalier et par les professionnels de santé mais, plus encore, largement ignoré par les patients et le public belge. L'information sur l'existence même de droits accordés aux patients et de médiateurs droit des patients compétents reste largement défaillante, à l'instar de ce qui a été montré par plusieurs travaux et enquêtes, notamment par l'Union Nationale des Mutualités Socialistes et le Centre de recherche et d'information des organisations de consommateurs (2007). Or, la plainte en tant qu'opérateur de justice n'a de possibilités concrètes de se déployer que si les droits font l'objet d'une information et d'une communication (tant à l'hôpital qu'à l'extérieur) qui permettent aux principes et aux normes d'être partagés dans le domaine public et ensuite d'être évoquées, mobilisées dans l'action - avec une visée d'empowerment ou de capacitation individuelle et collective (Périlleux \& Cultiaux, 2007 ; Genard \& Cantelli, 2010).

Durant nos enquêtes de terrain en Belgique, la plupart de nos contacts (accueil, infirmiers, docteurs, médiateurs interculturels) étaient embarrassés et gênés de ne pas pouvoir situer le médiateur droit des patients. Certes, tout droit une fois édicté nécessite un ample travail pédagogique et politique pour lui assurer un accomplissement pratique. Toutefois, le cas du droit des patients a ceci de singulier qu'il ne fait pas l'objet «d'investissements de formes » (Thévenot, 1986) conséquents, à savoir un ensemble de conventions, principes, instruments et acteurs (tels des syndicats ou des associations de patients) en mesure d'agir et de veiller au respect des droits du patient. Le point suivant montrera que les médiateurs droits du patient ne sont pas mieux outillés pour garantir ces droits et assumer une politique.

Ensuite, la dimension d'innovation sociale. Le caractère faiblement institutionnalisé du média- 
teur droit des patients et le rôle prépondérant de la direction médicale limitent l'ampleur et la portée de l'innovation dans le contexte politique belge. L'État octroie un budget à chaque hôpital ayant pour affectation la rémunération et le fonctionnement du service de médiation « droits du patient », soit l'équivalent d'une rémunération d'un travail mi-temps. Il y a quelques médiateurs temps plein, mais $74 \%$ occupent une autre fonction au sein de l'hôpital (assistant social, juriste, service infirmier, coordinateur qualité, etc.).

Le médiateur « droit des patients » peut intervenir à un niveau plus préventif mais sans avoir de compétences d'enquête (contrairement aux Commissaires locaux aux plaintes et à la qualité des services dans les hôpitaux au Québec, par exemple). Il est en mesure de mener un travail de prévention des plaintes via la sensibilisation de ses collègues, la diffusion de l'information, l'organisation de séminaires, etc. Un arrêté royal du 19 mars 2007 va uniformiser et autonomiser l'environnement proche du médiateur « droit des patients » en précisant quelques règles de bon fonctionnement : ligne téléphonique personnelle, adresse électronique propre, répondeur indiquant les heures de permanence, espace de réception approprié, etc.

Toutefois, l'importance de la direction médicale en Belgique reste prégnante. Il y a donc deux chemins possibles pour la plainte du patient. La direction peut décider de traiter des plaintes comme elle peut aussi déléguer des missions, en tout ou partie. Sauf cas exceptionnel, la direction de l'hôpital répond aux plaintes des patients en suivant ce type de protocole : elle réagit à un courrier écrit, et non pas à un appel téléphonique, à une rencontre inopinée ou à un rendez-vous ; elle juge et évalue la portée du courrier reçu, si le cas le nécessite en contactant les professionnels visés ou en transférant le courrier à d'autres services ou aux experts de l'assurance de l'établissement ; elle répond par écrit en exposant les motifs et en examinant la responsabilité des professionnels visés dans ce qui sera qualifié, requalifié ou disqualifié de problème, d'accident ou d'erreur.

La direction traite donc la voix des patients insatisfaits, qui peut aller d'une demande d'information, d'une plainte sur les repas, ou sur les sols glissants à une demande de dédommagement ou menace de procès. Et la direction peut, contrairement au médiateur, répondre à la plainte en faisant un geste vers le patient, un geste administratif et financier par exemple, en prenant en charge les frais d'examen ou d'hospitalisation. La loi ne prévoit pas que le médiateur droit des patients intervienne, d'une manière ou l'autre, dans ce processus. Les informations à ce sujet ne doivent pas lui être communiquées et ne sortent donc pas de la direction de l'hôpital. De la même manière, la direction peut décider de donner à son médiateur d'autres missions en interne, voire de lui donner, chose rare, un temps plein en tant que médiateur.

Enfin, les instruments d'évaluation. Les rapports des médiateurs sont mis en commun et traités comme une source d'information sur les dispositifs de médiation et sur la loi. Mais les défaillances dans cette mise en rapport sont étayées : "Le rapport du SPF [Service Public Fédéral] montre que de nombreux problèmes subsistent en matière de traitement des données mais, plus fondamentalement, sur le type même de 
données à traiter.» Cette première étape de mise en rapport est problématique en Belgique : les médiateurs y indiquent des informations parfois différentes et certains médiateurs élaborent un rapport pour plusieurs hôpitaux. La Commission a reçu 140 rapports annuels pour 144 hôpitaux généraux - et 66 pour les hôpitaux psychiatriques. Comment mettre en commun des informations et des chiffres alors que les acteurs concernés ne s'accordent pas a minima sur ce qu'est et sur ce que fait un médiateur dans un hôpital ? Les instruments législatifs et leur faible qualité de standardisation (Thévenot, 2009; Tournay, 2007 ; Timmermans \& Berg, 2003) des compétences des intervenants ouvrent le jeu à l'infini. Ces standards émoussés contribuent à éclater localement les formes possibles de la coordination.

Cela révèle combien ce travail de mise en commun repose sur un socle politique et cognitif préalable qui fait défaut; le cas belge montre que l'on ne s'accorde pas sur une définition - même minimale - uniforme des situations, des compétences et du contexte.

L'Association francophone des Médiateurs des Institutions de Soins, créée en 2005 (regroupant 45 médiateurs en Belgique francophone sur un total de 137), cherche à combler cette hétérogénéité par divers moyens (forums, réunions, séminaires, formation, etc.). Ceci étant, plusieurs aspects en relation avec l'incompatibilité de la fonction ont été réformés en Belgique. Ainsi, l'arrêté royal du 19 mars 2007 stipule qu'on ne peut plus exercer une fonction de cadre dans l'hôpital et être médiateur droit des patients - certains directeurs étaient, dans un passé proche, également médiateurs droit de patients.

\section{Conclusions sur l'impossible constitution d'un problème public}

Les plaintes des patients en Belgique forment donc un droit et figurent une politique mais qui se caractérisent par des repères fuyants, des appuis fragiles et de gages incertains. Pour conclure, nous voudrions avancer deux pistes provisoires et complémentaires susceptibles d'analyser plus avant les ressorts problématiques de ce droit et de cette politique.

D’abord, les Mutualités sont considérées en Belgique comme les garants du droit des patients, en tout cas de leurs affiliés. Pour rappel, elles font partie intégrante du modèle social belge de concertation et illustre le rôle des piliers (socialiste, catholique et libéral) dans la société belge. Elles défendent les intérêts des patients dans plusieurs organismes de sécurité sociale en Belgique. Et disposent d'un service juridique et médical, capable d'informer le patient notamment sur les problèmes de facturation et d'évaluation de la responsabilité lors d'accidents médicaux. Le caractère structurellement inabouti de la plainte et symétriquement celui de la médiation (comme en témoigne le cheminement tortueux du réseau professionnel des médiateurs droit des patients) relié à l'institutionnalisation des Mutualités en tant que défenseur des patients. Les dispositifs autour des « plaintes de basse intensité » telles que visées par la loi sur le droit des patients paraissent faiblement équipés et institutionnalisés voire sous-investis au profit du traitement des plaintes plus lourdes relatives à des litiges et accidents (impliquant assurances, Mutualités et tribunaux). La loi sur le fonds d'indemnisation des accidents médicaux, votée en mars 2010, atteste de cette 
attention publique sur les plaintes plus lourdes.

Ensuite, la faible présence des acteurs de la société civile (associations, collectifs, réseaux de patients) et leur intégration limitée dans les dispositifs de gestion des plaintes impliquent la constitution problématique d'un concernement et la difficile formation de problèmes publics au sujet des plaintes et du droit des patients dans la société belge. Même si les associations de patients sont représentées dans plusieurs organismes (notamment au sein de la Commission fédérale droit du patient) et qu'elles se sont regroupées au sein de la Ligue des usagers de services de santé en 1999, elles restent largement spécialisées autour d'une pathologie et sont généralement de taille modeste. Ainsi on perçoit mieux les ressorts variés d'un travail politique qui parvient difficilement à faire exister le droit de plainte des patients comme un problème public. Toutefois, la Belgique a connu plusieurs scandales, affaires et procès liés aux décès de patients. Ces événements ont contribué à réactiver la critique de la médecine et la critique des médecins, en particulier depuis 1996 avec la naissance de l'association Erreurs médicales créée par le père d'un enfant décédé suite à une erreur médicale. Pourtant, à suivre chacune de ces affaires dans la presse écrite, c'est davantage l'attribution et la reconnaissance de la responsabilité du médecin qui est thématisée plutôt que la plainte des patients en tant que droit et politique qui ne paraissent pas s'exprimer en tant que tels.

\section{$R \cdot \dot{E} \cdot F \cdot \dot{E} \cdot R \cdot E \cdot N \cdot C \cdot E \cdot S$}

J. ALLSOP, « Two sides of every story: complainants' and doctors' perspectives in disputes about medical care in general practice setting ", in Law and Policy, 16 (2), 1994, pp. 149-184.

C. BOSK, Forgive and remember. Managing medical failure, Chicago, University of Chicago Press, 2003 [1979].

M. CLEMENT et E. GAGNON, « The Quebec complaint examination system: stakeholder perspectives on the purpose and intake of complaints", in Health Care Analysis, (14) 2006, pp. 51-63.

N. DODIER, L'expertise médicale. Essai de sociologie sur l'exercice du jugement, Paris, Métailié, 1993.

O. FOLI, Plaintes, normes et intégration. Le cas d'une organisation bureaucratique, Thèse de doctorat en sociologie, Université Paris Dauphine, 2008.

J-L. GENARD et F. CANTELLI, «Pour une sociologie politique des compétences», in Politiques Sociales, 2010, pp. 103-120.

J-F. LAE, L'instance de la plainte. Une histoire politique et juridique de la souffrance, Paris, Descartes, 1996.

P. LASCOUMES, «L'usager dans le système de santé : réformateur social ou fiction utile?», in Politique et management public, 15-2, 2007, pp. 129-144.

M. MILLMAN, The unkindest cut: life in the backrooms of medicine, New York, Morrow Quill Paperbacks, 1977.

L. MULCAHY, Disputing doctors. The sociolegal dynamics of complaints about medical care, Maidenhead, Open University Press, 2003. 
S. NETTLETON et G. HARDING, « Protesting patients: a study of complaints submitted to a family health service authority », in Sociology of Health \& Illness, 16 (1), 1994, pp. 38-61.

J. PENEFF, Les malades des urgences, Paris, Métailié, 2000.

T. PERILLEUX et J. CULTIAUX, « Être responsable de sa santé ? Faces, accents et limites d'un argumentaire de responsabilisation des patients », in F. CANTELLI et J-L. GENARD (ed.), Action publique et subjectivité, Paris, LG.D.J, vol. 46, 2007, pp. 133-146.

I. PRESS, « Why people sue: sociological determinants of malpractice suits within hospitals», Law, Medicine and Health Care, vol. 12, 1984, pp. 53-62.

B. SINGER, Operative rights, Albany, State university of New York, 1993.

L. THEVENOT, «Governing life by standards», in Social Studies of Science, 39 (5), pp.793-813. L. THEVENOT, « Les investissements de forme ", in Thévenot L., Conventions économiques, Paris, PUF, 1986, pp. 21-71.

V. TOURNAY (ed.), La gouvernance des innovations médicales, Paris, PUF, 2007.

S. TIMMERMANS et M. BERG, The Gold Standard: the challenge of evidence-based medicine and standardization in health care, Philadelphia, Temple University Press, 2003.

Union Nationale des Mutualités Socialistes et Centre de recherche et d'information des organisations de consommateurs, Médiation hospitalière: perceptions et attentes des patients, Bruxelles, 2007.

P. VANOMERLINGEN, « La médiation des plaintes en milieu hospitalier. Expérience de la médiation locale », in G. Schamps (Ed.), Evolution des droits du patient, indemnisations sans faute des dommages liés aux soins de santé : le droit médical en mouvement, Paris, L.G.D.J, 2008, pp. 251-257.

M-N. VERHAEGEN, « La gestion de plaintes de patients auprès des services de médiation », in $\mathrm{G}$. Schamps (Ed.), Évolution des droits du patient, indemnisations sans faute des dommages liés aux soins de santé : le droit médical en mouvement, Paris, L.G.D.J, 2008, pp. 259-297.

R. YAJIMA et K. TAKAYANAGI, « The japanese health care system. Citizen complaints and citizen possibilities », in Handbook of international healthcare systems, éd. Thai, Khi V., Edward T. Wimberley, Sharon M. McManus, New York, Marcel Dekker, 2002, pp. 457-483. 
